\title{
Comparisons of eccentric binary black hole simulations with post-Newtonian models
}

\author{
Ian Hinder, ${ }^{1}$ Frank Herrmann, ${ }^{2}$ Pablo Laguna, ${ }^{3}$ and Deirdre Shoemaker ${ }^{3}$ \\ ${ }^{1}$ Max-Planck-Institut für Gravitationsphysik, Albert-Einstein-Institut, Am Mühlenberg 1, D-14476 Golm, Germany \\ ${ }^{2}$ Department of Physics, University of Maryland, College Park, MD 20742, USA \\ ${ }^{3}$ Center for Relativistic Astrophysics, School of Physics, \\ Georgia Institute of Technology, Atlanta, GA 30332, USA
}

\begin{abstract}
We present the first comparison between numerical relativity (NR) simulations of an eccentric binary black hole system with corresponding post-Newtonian (PN) results. We evolve an equalmass, non-spinning configuration with an initial eccentricity $e \approx 0.1$ for 21 gravitational wave cycles before merger, and find agreement in the gravitational wave phase with an adiabatic eccentric PN model with 2 PN radiation reaction within 0.1 radians for 10 cycles. The NR and PN phase difference grows to 0.7 radians by 5 cycles before merger. We find that these results can be obtained by expanding the eccentric PN expressions in terms of the frequency-related variable $x=(\omega M)^{2 / 3}$ with $M$ the total mass of the binary. When using instead the mean motion $n=2 \pi / P$, where $P$ is the orbital period, the comparison leads to significant disagreements with NR.
\end{abstract}

\section{INTRODUCTION}

Tremendous progress towards detecting gravitational waves is being made by observational efforts such as LIGO, VIRGO and GEO600. Just recently, LIGO has reached its designed sensitivity and is currently undergoing enhancements to increase the sensitivity by an order of magnitude as a step towards advanced LIGO. In anticipation of these enhancements, it is essential to have models of gravitational waveforms for all sources of gravitational radiation, in particular for binary black hole systems, since they are expected to be one of the most promising sources [1 [5].

Constructing these waveforms is a non-trivial task. Generating a complete waveform involves numerically solving the full Einstein equations in order to correctly describe the last few orbits and merger. This is computationally intensive with simulations running for weeks to produce a single accurate waveform. Furthermore, the parameter space of merging black hole binaries is quite large. In addition to the intrinsic black hole parameters (masses, spin magnitudes and orientations), there are the orbital parameters (eccentricity and semimajor axis). Because of the computational cost of producing numerical waveforms, the only way to have a hope of covering the parameter space efficiently is to use waveforms that combine NR solutions with results from the PN approximation. To achieve this goal, it is firstly important to cross-check the two methods to ensure that they give compatible results where $\mathrm{PN}$ is valid, namely for large enough binary separations. Secondly, it is necessary to investigate how close to the merger one can use the PN results (a recent study [6] addresses this question in the extreme-mass-ratio case using the theory of optimal asymptotic expansions).

Due to recent advances [7-9] in the field of numerical relativity, long-term accurate and stable evolutions of binary black hole systems spanning several orbits as well as the merger are now possible. The initial separations of the black holes in these simulations are now sufficient to make comparisons with waveforms generated in the PN approximation. Such comparisons have been made for equal-mass non-spinning [10 17], unequal-mass [18], and spinning 19 binaries, all in quasi-circular orbits. As a result, hybrid waveforms for quasi-circular orbits have been constructed which combine the PN waveform, accurate when the black holes are far apart, with the late inspiral and merger waveform that can only be obtained using full NR 20 23.

In this paper, we take the next step and extend for the first time the NR and PN comparison to the case of eccentric binary black hole systems. Only recently the first NR studies of bound eccentric binary black hole systems have been performed [24, 25], where the dependence of the final black hole mass and spin on the initial eccentricity for non-spinning, equal-mass systems was studied. It has long been known that far-separated eccentric binary systems emitting gravitational radiation will circularize [26]. However, it was not known what would happen if a binary black hole system still had significant eccentricity in the late stages of inspiral. Rather than forming a final black hole with a higher or lower spin, the NR results showed that even up to an initial eccentricity of $e \sim 0.4$, the final black hole mass and spin were the same as in the circular case, indicating that the rate of loss of eccentricity was sufficient for the binary to circularize prior to or during merger. Due to the tendency of eccentric binary systems to circularize, most of the expected astrophysical binary black hole sources for Earth-based gravitational wave detectors will have lost all their eccentricity by the time their waves enter the frequency band of the detector. However, several astrophysical scenarios have been proposed in which binary black hole systems in eccentric orbits might be detectable, for which it will be necessary to understand the dynamics and waveforms of eccentric binary black hole systems.

One such scenario may occur in the dense cores of globular clusters, where interactions between pairs of binary black hole systems eject one of the black holes, resulting in a stable hierarchical triple. This is a three-body system (three-body black hole systems have also been studied re- 
cently in NR 27]) consisting of two closely bound black holes and a third orbiting the center of mass of the first two. When the two orbital planes are strongly tilted with respect to each other, tidal forces from the third body can cause an orbital resonance, increasing the eccentricity of the inner binary. This is known as the Kozai mechanism [28]. It has been suggested [29] that this could lead to eccentricities greater than about 0.1 at the time the binary enters the frequency band of advanced ground-based detectors, followed by merger driven by gravitational radiation reaction. Stellar mass black hole binaries in globular clusters are expected to have a thermal distribution of eccentricities [30], and intermediate mass black holes in globular clusters are expected to have eccentricities between 0.1 and 0.2 while they are in the frequency band of the LISA detector [31]. Supermassive black holes are also sources for LISA, and it is currently unknown what eccentricity they might have [32]. They could potentially merge within the Hubble time from highly eccentric orbits if the Kozai effect was occurring [33]. It has also been shown that massive black hole binaries in disks of gas can merge without losing eccentricity if the disc is rotating in the opposite sense to the binary orbit 34]. Being able to measure the non-zero eccentricity from the waveforms will tell us about the physics of the system, and may also have implications for detection if quasi-circular templates are used.

The energy and angular momentum fluxes from the gravitational waves emitted by a comparable mass eccentric binary were originally determined by Peters and Mathews [26, 35] in the Newtonian limit. By balancing the time-averaged far-zone fluxes of energy and angular momentum with the loss of binding energy and angular momentum in the orbit, the rate of decay of the orbital semimajor-axis and eccentricity could be determined in the adiabatic approximation. The result showed that the eccentricity of a binary reduces by approximately a factor of three when the semimajor axis is halved.

The next order corrections to this result were obtained to $1 \mathrm{PN}$ and 1.5 PN order, enabling the study of the evolution of the orbital elements using the quasi-Keplerian parametrization of the orbit 36 [40]. With the use of a generalized Keplerian representation [41 43], this work was extended to $2 \mathrm{PN}$ [44, 45].

An improved method of variation of constants has been developed [46, 47] in order to construct models which for the first time go beyond the adiabatic approximation. Very small oscillations in the orbital elements were found on the timescale of the orbital period. The conservative 3 PN dynamics of an eccentric system in the quasi-Keplerian representation have been derived [48]. Recently, the complete $3 \mathrm{PN}$ energy and angular momentum fluxes have been determined [49 52 ].

The availability of the energy flux to $3.5 \mathrm{PN}$ order [53] in the quasi-circular case has led to successful matches with NR waveforms, with agreement in the waveform phase within 0.05 radians between 30 and 15 cycles before merger, and within several radians up to the merger 15] for some PN models, though the level of agreement near merger is model dependent. The TaylorT4 model, specifically, agrees within 0.05 radians up to $M \omega_{\mathrm{gw}}=0.1$. Recently, it has been shown that for TaylorT4, the energy flux is identifiably different from the NR result even 25 cycles before merger [17].

A circular binary black hole inspiral gives rise to waveform dynamics which are in some sense simple: the amplitude and frequency increase monotonically, which may explain why the adiabatic approximation works so well. Eccentric orbits on the other hand give rise to waveforms with oscillations in the amplitude and frequency, and comparison with NR in this case will provide a significantly more stringent test of the PN approximation.

In this paper, we present the first analysis of the agreement between PN and NR eccentric waveforms. We restrict to the equal-mass, non-spinning case. We use the 3 PN conservative quasi-Keplerian orbit equations [48], combined with the $2 \mathrm{PN}$ evolution of the orbital elements [46] to construct adiabatic PN waveforms, determined by four independent initial parameters. We then present a full NR evolution which starts 21 gravitational wave cycles before merger with an estimated initial eccentricity of $e \approx 0.1$. We assume that the NR simulation gives the final stage of a full waveform, such as one that would be observed in nature. We then choose a fitting interval in time and use least squares fitting to find the parameters of the PN waveform which best matches the numerical data in that interval. We find agreement between the NR and PN gravitational wave phase within 0.1 radians for 10 wave cycles at the start of the simulation. The NR and PN phase difference grows to 0.6 radians 5 cycles before merger, corresponding to $M \omega_{\mathrm{gw}}=0.1$.

As has been previously shown in the circular case, we find that different PN approximants lead to different levels of agreement with NR [15]. We show here that an eccentric PN model expanded in terms of the mean motion $n=2 \pi / P$, where $P$ is the orbital period, leads to significant disagreements with $\mathrm{NR}$, whereas using the frequency-related variable $x=((2 \pi+\Delta \phi) / P)^{2 / 3}$, where $\Delta \phi$ is the precession angle per period, gives much better agreement.

In Section $\amalg A$ we describe the eccentric PN model we will use in our comparisons. The PN expressions are given in outline form to make clear precisely how we are constructing the solutions; the full expressions are given in the appendix. In Section [IB, we describe the methods used in our NR simulations that have not previously been described; specifically, we discuss the method of constructing initial data parameters with eccentricity $e \approx 0.1$. We present the results of the numerical simulations in Section IIIA, along with an analysis of the errors. In Section IIC, we describe the method we use for matching NR and PN waveforms. Section IIIB contains the main result of this paper, which is the comparison of the PN and NR solutions. Finally, we discuss the consequences of the results and our plans for future work in Section IV 


\section{METHODS}

\section{A. Eccentric post-Newtonian model}

We first review the solution of eccentric Newtonian orbits, in order to fix notation and to illustrate our general method for solving the PN system. For a detailed treatment, see for example Ref. [54] 1]. The system under consideration consists of two point particles of masses $m_{1}$ and $m_{2}$. The total mass is $M=m_{1}+m_{2}$. We will use $M$ as the mass scale for all numerical quantities in our NR simulations, and work in units in which $G=c=1$. The reduced mass is $\mu=m_{1} m_{2} / M$ and the symmetric mass ratio is $\eta=\mu / M$. We will give expressions for arbitrary mass ratios $\eta$, although in this work we will only be considering equal-mass systems, for which $m_{1}=m_{2}=M / 2$, $\mu=M / 4$ and $\eta=1 / 4$. For Newtonian orbits, the energy $E$ and angular momentum $J$ are constants of the motion and can be expressed in terms of the mean motion $n$ and the eccentricity $e$. The conservation of $J$ means that the orbit is restricted to a plane. The mean motion is related to the orbital (pericenter to pericenter) period $P$ and the semimajor axis $a$ by $n=2 \pi / P=a^{-3 / 2} M^{1 / 2}$. In the Newtonian case, the pericenter occurs at the same value of the relative angular coordinate $\phi$ on each orbit; i.e. there is no precession. There is no closed form solution for the relative orbital radius $r$ or angular frequency $\dot{\phi}$ in terms of time, but they can be expressed in terms of the eccentric anomaly $u$,

$$
\begin{aligned}
& r=a[1-e \cos u] \\
& \dot{\phi}=\frac{n \sqrt{1-e^{2}}}{[1-e \cos u]^{2}} .
\end{aligned}
$$

The eccentric anomaly $u$ satisfies Kepler's equation,

$$
l=u-e \sin u
$$

where the mean anomaly $l$ is given by $i=n$. Since $n$ is a constant, we can integrate to obtain $l=n\left(t-t_{0}\right)$ and Eq. (3) is a transcendental algebraic equation for $u$, which can be solved numerically, for example by Newton's method, at each $t$. Thus we can obtain $r$ and $\dot{\phi}$ (and hence $\dot{r}$ and $\phi$ ) at any time $t$. Each orbit is parametrized by the constants $n, e, \phi_{0} \equiv \phi\left(t_{0}\right)$ and $l_{0} \equiv l\left(t_{0}\right)$.

The Newtonian system is conservative in the sense that it admits a conserved energy and angular momentum, which can be expressed in terms of the constants $n$ and $e$. One can also derive conservative equations in the $\mathrm{PN}$ case; the Newtonian equations for $r, \dot{\phi}$ and $l$ are modified by the addition of higher order (in $n$ ) terms. In the PN case, the quasi-Keplerian parametrization leads to three eccentricities, $e_{t}, e_{r}$ and $e_{\phi}$, representing deviations from

\footnotetext{
1 Note that in Ref. [54], the eccentric anomaly is called $\psi$ rather than $u$, and the period $T$ rather than $P$. The notation used in this work reflects that commonly used in the PN literature.
}

circular motion in $t, r$ and $\phi$, but these are related to each other by PN equations and it is sufficient to consider just $e_{t}$. To Newtonian order, all three are equal.

In the conservative $\mathrm{PN}$ equations, $n$ and $e_{t}$ are still constants, but the orbits precess. Note that the period $P$ of the orbit is defined to be the time from pericenter to pericenter, and due to the effects of precession, this is not the time to go from angular coordinate $\phi$ to $\phi+$ $2 \pi$. The angle of precession of the pericenter during one (pericenter to pericenter) orbit of period $P$ is denoted $\Delta \phi$. Following Refs. [49, 50], we define

$$
\omega \equiv \frac{2 \pi+\Delta \phi}{P}
$$

to be the angle swept out by the orbit from pericenter to pericenter in one period $P$. Note that in the conservative $\mathrm{PN}$ system, this is a constant. In the circular case, where $\dot{\phi}$ is a constant, we have $\omega=\dot{\phi}$. We will investigate two different PN models which differ only in the choice of variable used (and hence in higher order uncontrolled remainder terms). In Ref. [47], the eccentric system is described in terms of the mean motion $n$ and the eccentricity $e_{t}$. We present the equations here in terms of the variable $x \equiv(M \omega)^{2 / 3}$ and $e_{t}$. We call the two resulting PN models the $x$-model and the $n$-model. See Sec. IIIC for more discussion of these two models.

We now give the $3 \mathrm{PN}$ conservative orbital dynamics; we work throughout in modified harmonic coordinates, in which these expressions have been derived [48]. The $3 \mathrm{PN}$ conservative dynamics were first determined in Ref. [48], and were written out explicitly in terms of $n$ and $e_{t}$ in Ref. 47]. Here, for brevity, we will omit lengthy high order PN expansions; the full expressions are available in the appendix. The abbreviated forms of the conservative dynamics, in terms of $x$ and $e_{t}$, are

$$
\begin{aligned}
r / M= & {\left[1-e_{t} \cos u\right] x^{-1}+r_{1 \mathrm{PN}}+r_{2 \mathrm{PN}} x } \\
& +r_{3 \mathrm{PN}} x^{2}+\mathcal{O}\left(x^{3}\right) \\
M \dot{\phi}= & \frac{1 \sqrt{1-e_{t}^{2}}}{\left[1-e_{t} \cos u\right]^{2}} x^{3 / 2}+\dot{\phi}_{1 \mathrm{PN}} x^{5 / 2}+\dot{\phi}_{2 \mathrm{PN}} x^{7 / 2} \\
& +\dot{\phi}_{3 \mathrm{PN}} x^{9 / 2}+\mathcal{O}\left(x^{11 / 2}\right) \\
l= & u-e_{t} \sin u+l_{2 \mathrm{PN}} x^{2} \\
& +l_{3 \mathrm{PN}} x^{3}+\mathcal{O}\left(x^{4}\right) \\
M \dot{l}= & M n=x^{3 / 2}+n_{1 \mathrm{PN}} x^{5 / 2}+n_{2 \mathrm{PN}} x^{7 / 2} \\
& +n_{3 \mathrm{PN}} x^{9 / 2}+\mathcal{O}\left(x^{11 / 2}\right)
\end{aligned}
$$

where the quantities $r_{1 \mathrm{PN}}, \dot{\phi}_{1 \mathrm{PN}}, \ldots$ are functions of $e_{t}$ and $u$, but $n_{1 \mathrm{PN}}, \ldots$ are functions only of $e_{t}$. Since the right hand side of Eq. (8) is given in terms of the constants $x$ and $e_{t}$, it can be trivially integrated to give $l(t)$ in terms of an integration constant $l_{0}$ at some $t_{0}$. So given constants $x, e_{t}$ and $l_{0}$, we can solve Eq. (77) numerically for $u$ at each $t$ by root-finding, then insert $u$ into Eqs. (56) to obtain the coordinate motion of the conservative $3 \mathrm{PN}$ system. 
The conservative system is expected to be a good approximation on timescales over which the energy and angular momentum lost to gravitational radiation is negligible. To go beyond this approximation, we will model these losses adiabatically; i.e. they will be averaged over the orbital period. The losses are derived by computing the gravitational wave energy and angular momentum flux at infinity and equating the energy and angular momentum radiated to that lost from the system. The equations for $\dot{E}$ and $\dot{J}$ can be used to derive equations for $\dot{x}$ and $\dot{e}_{t}$. The equations to $2 \mathrm{PN}$ order are given in Ref. [47] in terms of $n$ and $e_{t}$. In terms of $x$ and $e_{t}$, we have

$$
\begin{aligned}
M \dot{x}= & \frac{2 \eta}{15\left(1-e_{t}^{2}\right)^{7 / 2}}\left(96+292 e_{t}^{2}+37 e_{t}^{4}\right) x^{5}+\dot{x}_{1 \mathrm{PN}} x^{6} \\
& +\dot{x}_{1.5 \mathrm{PN}} x^{13 / 2}+\dot{x}_{2 \mathrm{PN}} x^{7}+\mathcal{O}\left(x^{15 / 2}\right) \\
M \dot{e}= & \frac{-e \eta}{15\left(1-e_{t}^{2}\right)^{5 / 2}}\left(304+121 e_{t}^{2}\right) x^{4}+\dot{e}_{1 \mathrm{PN}} x^{5} \\
& +\dot{e}_{1.5 \mathrm{PN}} x^{11 / 2}+\dot{e}_{2 \mathrm{PN}} x^{6}+\mathcal{O}\left(x^{13 / 2}\right)
\end{aligned}
$$

Since the evolution is treated adiabatically, the functions $\dot{x}_{1 \mathrm{PN}}, \dot{e}_{1 \mathrm{PN}}, \ldots$ depend only on $e_{t}$, and not on $u$. Hence, the adiabatic evolution equations for $x$ and $e_{t}$ form a closed system, and can be solved independently of the Kepler equation. Given initial conditions $x(0)$ and $e_{t}(0)$, we can solve the system of ODEs numerically to obtain $x(t)$ and $e_{t}(t)$.

In the presence of time-varying $x$ and $e_{t}$, Eq. (8) must be integrated to obtain $l(t)$. The rest of the computation proceeds as in the conservative case; $u$ is determined numerically by root-finding in Eq. (7), and then $u, x$ and $e_{t}$ are inserted at each time into Eqs. (5] 6).

We use analytical expressions for the functions $r_{1 \mathrm{PN}}, \dot{\phi}_{1 \mathrm{PN}}, \ldots$ to obtain numerical solutions for $r$ and $\dot{\phi}$, but due to the complexity of the expressions for $\dot{r}$ and $\phi$, we choose to obtain $\dot{r}$ and $\phi$ by numerically differentiating and integrating the $r$ and $\dot{\phi}$ solutions respectively. This makes a difference to terms at higher $\mathrm{PN}$ orders that we are currently neglecting.

We have checked our expressions for $r$ and $\dot{\phi}$, as well as the 3 PN Kepler equation, by deriving them from the orbital elements in terms of $E$ and $h$ [48], and comparing with the explicit expressions in terms of $n$ and $e_{t}$ [47]. This completes the description of the coordinate motion.

Since the NR and PN solutions are in different coordinate systems, we must compare them using some coordinate-independent quantity. We will use the gravitational wave frequency; specifically the $\ell=2, m=2$ mode of the Newman-Penrose $\Psi_{4}$ quantity, as it is readily available from the NR simulations.

The complex PN waveform strain is given (to leading
Newtonian order) by

$$
\begin{aligned}
h= & h_{+}-i h_{\times} \\
h_{+}= & -\frac{M \eta}{R}\left\{( \operatorname { c o s } ^ { 2 } \theta + 1 ) \left[\cos 2 \phi^{\prime}\left(-\dot{r}^{2}+r^{2} \dot{\phi}^{2}+\frac{M}{r}\right)\right.\right. \\
& \left.\left.+2 r \dot{r} \dot{\phi} \sin 2 \phi^{\prime}\right]+\left(-\dot{r}^{2}-r^{2} \dot{\phi}^{2}+\frac{M}{r}\right) \sin ^{2} \theta\right\} \\
h_{\times}= & -\frac{2 M \eta}{R} \cos \theta\left\{\left(-\dot{r}^{2}+r^{2} \dot{\phi}^{2}+\frac{M}{r}\right) \sin 2 \phi^{\prime}\right. \\
& \left.-2 r \cos 2 \phi^{\prime} \dot{r} \dot{\phi}\right\},
\end{aligned}
$$

where $\phi^{\prime} \equiv \phi-\varphi$, and $\theta$ and $\varphi$ are the spherical polar angles of the observer. Eqs. 11,13 are taken from Ref. [45] but with the sign convention for the $\cos 2 \phi^{\prime}$ and $\sin 2 \phi^{\prime}$ terms of Refs. [46, 47]. Using only the leading (quadrupolar) contribution to $h$ is called the restricted waveform approximation. The strain $h$ can be decomposed into spin-weight $s=-2$ spherical harmonics, and the $\ell=2, m=2$ mode is given by

$$
\begin{aligned}
h^{22} & =\int_{-2} Y_{2}^{2^{*}}(\theta, \varphi) h(\theta, \varphi) d \Omega \\
& =-\frac{4 M \eta e^{-2 i \phi}}{R} \sqrt{\frac{\pi}{5}}\left(\frac{M}{r}+(\dot{\phi} r+i \dot{r})^{2}\right),
\end{aligned}
$$

where ${ }_{-2} Y_{2}^{2}(\theta, \varphi)=\frac{1}{2} e^{2 i \varphi} \sqrt{5 / \pi} \cos ^{4}(\theta / 2)$. We insert the coordinates, $\phi, \dot{\phi}, r, \dot{r}$, into Eq. (15) to obtain the $\ell=$ $2, m=2$ spin-weight $s=-2$ [55] mode of the waveform strain. Finally, using $\Psi_{4}^{22}=\ddot{h}^{22}$ we differentiate $h^{22}$ twice with respect to time to obtain the (complex) $\ell=2, m=2$ mode of $\Psi_{4}$. This is split into amplitude and phase, and undetermined additive multiples of $2 \pi$ in the phase are determined by continuity.

We have described one procedure for constructing PN eccentric waveforms. Note that this is not unique; different procedures will differ by the 'uncontrolled remainder terms' of higher PN order than we have considered. Specifically, we have chosen to solve the $2 \mathrm{PN}$ truncated adiabatic evolution equations for $x$ and $e_{t}$ numerically, rather than constructing an analytic expansion for the solution and then truncating it to $2 \mathrm{PN}$. This makes a difference to the solution in the circular case [11], and has been shown [15] to give better phase agreement with NR. In Ref. [15], the circular waveform constructed using this approach is named TaylorT4, and the waveform phase agrees significantly better with NR than the TaylorT1, TaylorT2 and TaylorT3 approximants. For simplicity, we have also limited the computation of the waveform as a function of the coordinates to Newtonian (quadrupolar) accuracy, and restrict our comparisons to the phase rather than the amplitude. Higher order corrections to the waveforms are available [44, 56], though not in a form which is convenient to use in this work. We have also chosen to construct some derivative quantities by numerical differentiation; where this is the case, we have verified 
that the effects of discretization on the resulting waveform phase are much smaller than any numerical errors we have in our NR simulations.

\section{B. Numerical relativity methods}

Our NR simulations are based on the moving punctures approach without excision [8, 9]. Initial data representing the binary black hole system is constructed with a conformally flat metric and Bowen-York extrinsic curvature, and the constraints are solved using the TwoPunctures [57] spectral code. The evolution in time is performed using our BSSN [58 60 finite differencing code generated using the Kranc [61] code generation package. The Cactus [62] infrastructure is used for parallelization, I/O and parameter handling, and for adaptive mesh refinement we use Carpet 63]. The code has been previously described in more detail 64], however we have since modified it to use sixth order spatial finite differencing as described in Ref. [65] in order to improve accuracy. We here use 9 levels of box-in-box mesh refinement, where the outermost (base) grid covers the domain $x^{i} \in[-384,384]$. On the outer boundary, a simple spherical outgoing wave boundary condition is applied to each variable as is conventional for finite differencing BSSN codes in NR (see Ref. [66] for more details). Formally, this boundary condition respects neither the constraints nor the characteristic structure of the equations. For very short simulations, it is possible to place the outer boundary far enough out that it is causally disconnected from the coordinate spheres on which the waveforms are computed, but for the long simulation we present here it is not computationally feasible to do this in our code. A discussion of the possible errors introduced can be found in Sec. IIIA.

The free parameters in the Bowen-York extrinsic curvature are the coordinate locations and linear momenta of the two black holes. We obtain these parameters using the conservative $3 \mathrm{PN}$ expressions for eccentric orbits [47]. These expressions require specification of the two constants, $e_{t}$ and $n$ (the eccentricity and mean motion). We choose $n=0.0156 / M$ and $e_{t}=0.1$, and compute the coordinate separation, $r$, from the $3 \mathrm{PN}$ expression in terms of $n$ and $e_{t}$, and use it in the BowenYork extrinsic curvature. The tangential linear momentum of each black hole at apocenter, $p_{y}$, is obtained from $J=p_{y} r$, where $J$ is computed as a PN expansion in $n$ and $e$. We solve iteratively for the base mass parameters to ensure that the irreducible masses of the black holes are $m_{1}=m_{2}=0.5 M$, where $M$ is a mass scale. As such, the mass scale $M$ is the sum of the irreducible masses of the black holes. This procedure results in initial coordinate locations $x_{ \pm}^{i}=( \pm 7.1570737463,0,0) M$, initial linear momenta $P_{ \pm}^{i}=(0, \pm 0.07191137095,0) M$, and initial bare masses $m_{\text {bare }}^{ \pm}=0.4903157830 M$. The resulting spacetime has $\mathrm{ADM}$ mass $M_{\mathrm{ADM}}=0.991413 M$.

This choice of initial data parameters has the follow- ing limitations. Firstly, only the conservative PN expressions have been used, which means that there is no consideration of the inspiral velocity. Secondly, there will be an error in the parameters due to the truncation of the PN series. Thirdly, the use of PN parameters (in this case in harmonic coordinates) directly substituted into the Bowen-York extrinsic curvature, assumes that the differences in the coordinate systems are small. We will see later that these initial data parameters agree reasonably well with the subsequent evolution.

\section{Fitting the post-Newtonian model to numerical relativity data}

We now discuss our method for determining a PN model which corresponds to our numerical simulation results. The PN approximation is very accurate when the binary system is far separated, becomes less accurate in the later stages of inspiral, and is no longer valid during some period leading up to merger. Using NR, we can simulate the late inspiral and merger. Ultimately, we would like to construct a waveform which most closely resembles one that would be observed in nature from early inspiral all the way through to merger. We will assume that the NR result gives the final part of this hypothetical full waveform, and use a PN waveform to approximate the full waveform before the start of the NR one. In this paper, we will not construct a hybrid waveform from the $\mathrm{PN}$ and NR results.

In this work, we will look for agreement in the gravitational wave frequency of the $\ell=2, m=2$ mode of $\Psi_{4}$,

$$
\omega_{\mathrm{gw}} \equiv \dot{\phi}_{\mathrm{gw}}=\frac{d}{d t} \arg \Psi_{4}^{22},
$$

as is common in the circular case. We will use the suffix ' $\mathrm{gw}$ ' to indicate that the quantity we are considering is related to the gravitational wave, and not the coordinate motion. We choose a time interval $\left[t_{1}, t_{2}\right]$ in the numerical simulation and use least squares fitting to determine the parameters of the PN model that best fits the numerical data in that interval. We will find in Section $\amalg I A$ that the black hole masses in the numerical simulations are essentially constant at $m_{1}=m_{2}=0.5 \mathrm{M}$ for the inspiral part of the simulation, so we do not fit for the masses when matching to PN. Thus, the eccentric PN model is determined uniquely by a choice of the functions $X, e_{t}$, $l$ and $\phi$ at a given time $t_{0}$, where $X=x$ or $n$ depending on the PN model being constructed. We define initial conditions

$$
y_{0} \equiv\left[X_{0}, e_{0}, l_{0}, \phi_{0}\right]
$$

and the residual

$$
Q\left(y_{0}\right) \equiv \frac{1}{N} \sum_{t \in I}\left[\omega_{\mathrm{PN}}\left(t ; y_{0}\right)-\omega_{\mathrm{NR}}(t)\right]^{2},
$$


using points $t$ from the numerical simulation in the interval $\left[t_{1}, t_{2}\right] . Q$ is then minimized numerically over $y_{0}$, where for each $y_{0}$, the $\mathrm{PN}$ equations must be solved to construct the waveform. The minimization requires an initial estimate of $y_{0}$. We find that using a local minimization method (for example, the principal axis method) can lead to inconsistent results. Specifically, the final fitted parameters show a dependence on the initial estimate due to the existence of local minima in the residual. Instead, we use a global minimization method, requiring an order of magnitude more iterations (typically around 5000), and hence increased computational resources. We find that minimization by the method of differential evolution, as implemented in Mathematica's NMinimize function, works well. A typical minimization for a given fitting window takes about 20 minutes on a laptop. Note that since the wave frequency $\omega_{\mathrm{gw}}$ is independent of $\phi_{0}$, in practice we determine $\phi_{0}$ by a separate least squares fit between the $\mathrm{PN}$ and $\mathrm{NR}$ waveform phases.

So, given a fitting interval, we can determine a $\mathrm{PN}$ model, identified by the parameters $y_{0}$. If the model and data matched exactly, the fitted parameters $y_{0}$ would be independent of the fitting interval. However, the errors in the PN approximation cause the fit to be imperfect. If these errors are large, the dependence on the fitting interval will be significant.

Once the parameters $y_{0}$ have been obtained, we can use these parameters to construct a final PN model, which will be the model that best approximates the full solution in the fitting interval.

\section{RESULTS}

\section{A. Numerical relativity simulation results}

In this section, we describe the results of our NR simulations, and analyze the numerical errors. The PN model gives the limiting form of the waveform at large distances from the source, whereas in the numerical code we compute the waveform on coordinate spheres of finite radii $r_{\text {ext }} / M=\{30,40, \ldots, 150\}$. We therefore extrapolate the waveform to infinite radius using the method described in Ref. [15]. To extrapolate the waveform, we first shift the waveform measured at each extraction radius in time by the estimated light propagation time to the extraction sphere, given by the Schwarzschild tortoise coordinate [67],

$$
r^{\star}=r_{\text {areal }}+2 M_{\mathrm{ADM}} \log \left(\frac{r_{\text {areal }}}{2 M_{\mathrm{ADM}}}-1\right),
$$

where we approximate $r_{\text {areal }} \approx r+M_{\mathrm{ADM}}$ (see Ref. [15] for further details; even if this relation does not hold exactly, the deviation will be included in our extrapolation error estimates). The amplitude and phase are then separately extrapolated by a least squares fit to an $n$th degree polynomial in $1 / r, f^{n}(r) \equiv f_{\infty}+\sum_{i=1}^{n} a_{i} / r^{i}$ at each time $t-r^{\star}$. We estimate the error in the $n$th order extrapolation as $e^{n} \equiv f^{n+1}-f^{n}$. We find that using extraction radii $r_{\text {ext }}=\{70,80, \ldots, 150\}$ in combination with first order extrapolation gives the best results. Using higher order extrapolation does decrease the error, but the extrapolant contains more noise.

We ran three simulations at different resolutions in order to assess the finite differencing error. The finest refinement boxes were coordinate cubes of side $1.24 M$ and consisted of $48^{3}, 64^{3}, 80^{3}$ points in the three runs. This leads to finest grid spacings of $h_{\mathrm{f}}=$ $M / 38.7, M / 51.6, M / 64.5$. To investigate the finite differencing error, we consider the convergence properties of the gravitational wave phase,

$$
\phi_{\mathrm{gw}}\left(t-r^{\star}\right) \equiv \arg \left[\Psi_{4}^{22}\left(t-r^{\star}\right)\right],
$$

extrapolated to infinite radius. In Fig. 1, we plot the convergence order of $\phi$. For $t-r^{\star}<500$, we see no clear convergence order, but the differences between the phases at the three resolutions are less than 0.01 radians. For $500<t-r^{\star}<2000$ we see a convergence order which drops from 6 to 5 , after which the order drops to about 1 for a small period around the merger. The fact that the convergence order is not clearly 6 may be explained by the fact that we have second, fourth and sixth order components in the simulation. Since we do not have clean sixth order convergence, we cannot reliably use Richardson extrapolation to obtain a more accurate result. However, we can use extrapolation of the highest two resolutions using the observed approximate convergence order of 5 to provide an estimate of the error in the solution. Note that for the time region we will use for matching with $\mathrm{PN}(t<1000)$, the convergence order of 5 is a good approximation. Figure 2 shows the finite differencing error estimate compared with the estimate of the error in the extrapolation to infinite radius. The dotted line represents the time of the peak in $\left|\Psi_{4}^{22}\right|$, which is a good indicator of the merger time. Note the sudden increase in the extrapolation error shortly after the merger. Also note that any significant effects arising from numerical reflections of the waves from refinement boundaries are expected to be covered by the finite differencing error bars, as these effects should diminish with increased resolution.

When comparing with PN later, we will add the errors from finite differencing and from extrapolation in quadrature to provide an estimate of the overall error in the numerical waveform. Note that the approximately exponential growth of the finite differencing error in Fig. 2 has been previously observed in the circular case 65 .

When comparing NR and PN models, we wish to use the gravitational wave frequency $\omega_{\mathrm{gw}}$. However, as seen before for both finite differencing [13] and pseudospectral [15] codes in the circular case, $\omega_{\mathrm{gw}}$ has noticeable high frequency error at early times when the amplitude of the radiation is low. In our case, this comes from numerical reflections of the initial spurious radiation, present in 


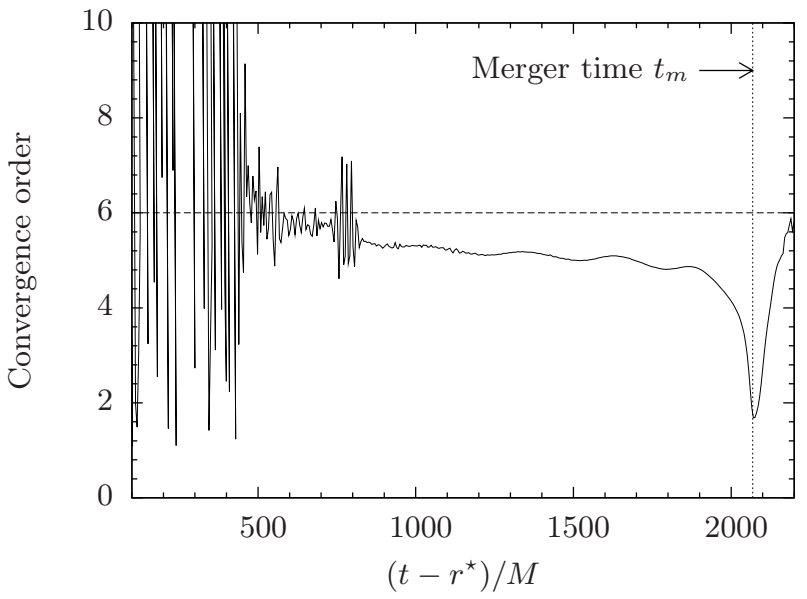

FIG. 1. Convergence order of the NR gravitational wave phase $\phi_{\mathrm{gw}}$. Deviations from the expected value of 6 may be caused by lower order components in the code.

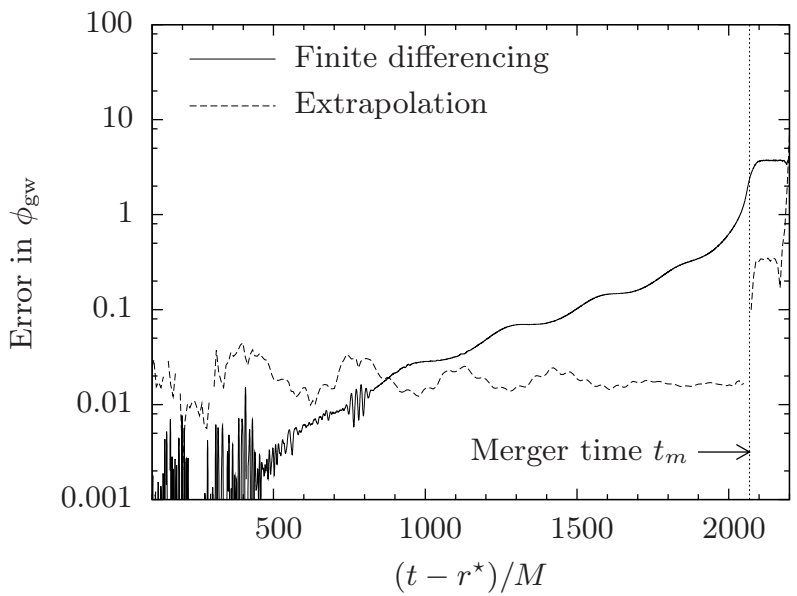

FIG. 2. Errors in the NR gravitational wave phase $\phi_{\mathrm{gw}}$ from the effects of finite resolution and extrapolation to infinite radius

the initial data, from mesh refinement boundaries. Since this is precisely the regime in which we would like to match with PN, this high frequency error must be removed. We find that this can be achieved very effectively by filtering the noisy region of $\omega_{\mathrm{gw}}$ in the Fourier domain. We first tried using a moving averages filter, but this tended to systematically reduce the amplitude of the oscillations in $\omega_{\mathrm{gw}}$, which we found unacceptable. We also chose not to fit a polynomial to $\omega_{\mathrm{gw}}$ as has been done in the circular case [13], due to the naturally oscillatory nature of the eccentric signal. To perform the filtering, we proceeded as follows. We first chose an interval of time, $\left[t_{1}, t_{2}\right]$, in which to perform the filtering. We chose $\left[t_{1}, t_{2}\right]=[80 \mathrm{M}, 1680 \mathrm{M}]$, excluding the late inspiral and merger as well as the initial spurious radiation from the filtering region. We then performed a discrete

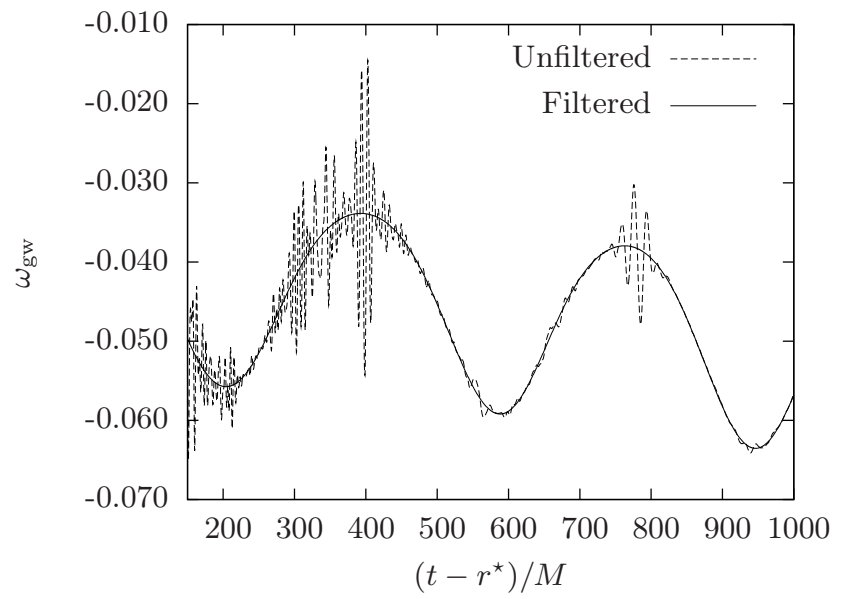

FIG. 3. Filtering of NR gravitational wave frequency in the Fourier domain. The solution in the region containing noise is truncated to the lowest 30 Fourier modes.

Fourier transform of the data, removed all but the lowest 30 modes, and then inverse transformed. We found that 30 modes were sufficient to represent the signal; this was judged by subtracting the filtered from the unfiltered signal, and observing essentially only noise. Taking only the first 30 modes corresponds to a frequency cutoff of $\omega_{\max }=30 \times 2 \pi /\left(t_{2}-t_{1}\right) \approx 0.1 M^{-1}$, or modes with a period of $T_{\min } \approx 50 M$. Note that this is not comparable to filtering the evolved variables or even $\Psi_{2,2}^{4}$; it is the frequency of $\Psi_{2,2}^{4}$ that is being filtered. Since the original signal is not periodic, Gibbs phenomena were observed as oscillations near the endpoints of the filtered region. We therefore removed a segment of length $80 M$ from the beginning and end of the filtered region before re-inserting the filtered region into the full signal. Fig. 3 shows the result of the filtering.

We have monitored the irreducible masses $M_{\text {irr }}$ of the apparent horizons in the lowest resolution simulation. The computed mass of each black hole drops from its initial value of 0.5 by only $2 \times 10^{-4} M$ by the time of the merger, and we ascribe this effect to finite differencing error. We have not computed horizons at higher resolutions due to computational expense. Thus, within our numerical errors, we do not detect any physical growth of the horizons during the inspiral, which potentially could have occurred due to absorption of gravitational wave energy in the initial part of the simulation, as has been studied in detail in previous work [68].

The spins of the black holes, as measured using an approximate technique derived from the isolated horizon formalism 69, 70], increase during the simulation to only $S^{z}=10^{-4} / M^{2}$ before the merger. This is independent of finite differencing resolution, but we expect this tiny spin to be of little consequence to the PN comparison, which does not contain the effects of spin.

The outer boundary in the simulations is at $x^{i}=$ $\pm 384 M$, and as mentioned in Sec $\amalg B$, the boundary con- 


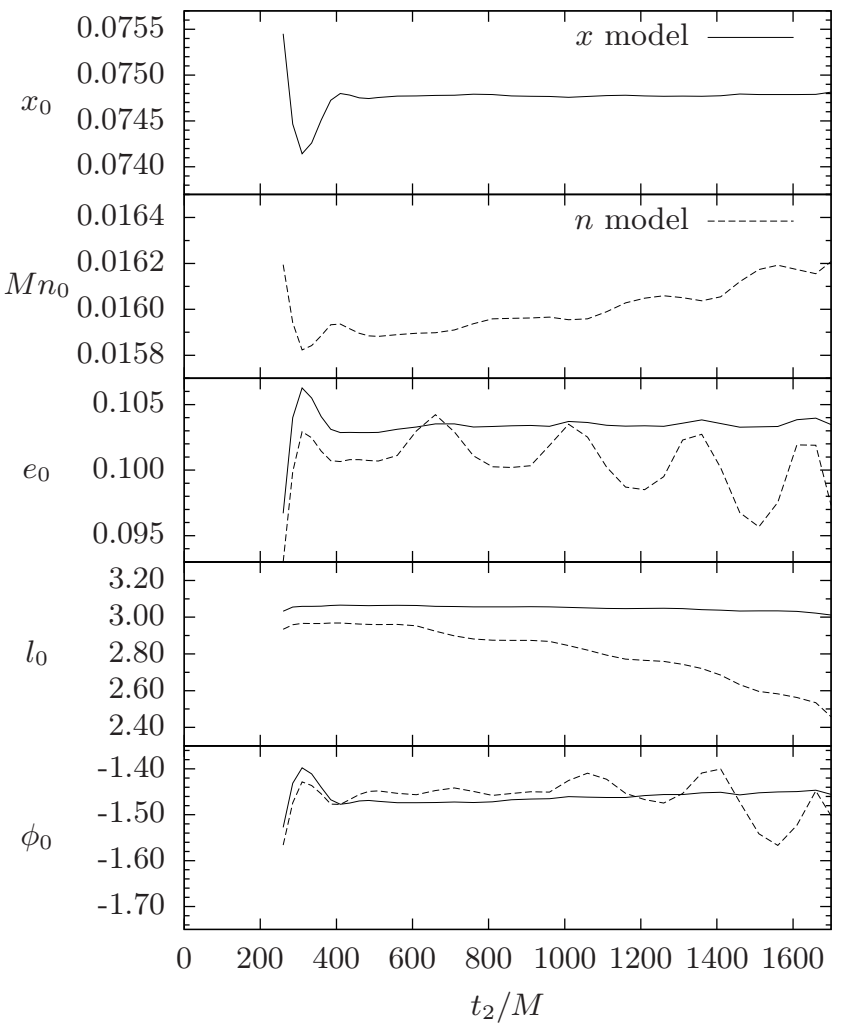

FIG. 4. PN parameters for the $x$ and $n$ models as determined from fitting windows $\left[t_{1}, t_{2}\right]$ for $t_{1}=210 M$ and various values of $t_{2}$.

dition is a source of error in the simulation. To measure the effect of this error, we have repeated the low resolution simulation, which has only modest computational cost, with the outer boundary moved to $x^{i}= \pm 768 M$ by enlarging the coarsest grid. We find that the effect on the waveform phase is much smaller than the estimated errors in the high resolution simulation due to finite differencing and extrapolation to infinite radius, and we conclude that the outer boundary is not a significant source of error in the simulation. In future, with more accurate simulations, this will need to be addressed further.

The simulations at the three different resolutions consumed approximately 5000, 11000 and 16000 CPU hours respectively, each one running on 32 cores of the LoneStar supercomputer.

\section{B. Comparing numerical relativity simulations with post-Newtonian models}

We now discuss the results of applying the fitting procedure described in Section IC to the numerical simulation results.

Figures 4 and 5 show the parameters $\left[x_{0}, e_{0}, l_{0}, \phi_{0}\right]$ (for the $\mathrm{x}$ model) and $\left[n_{0}, e_{0}, l_{0}, \phi_{0}\right]$ (for the $\mathrm{n}$ model) deter-

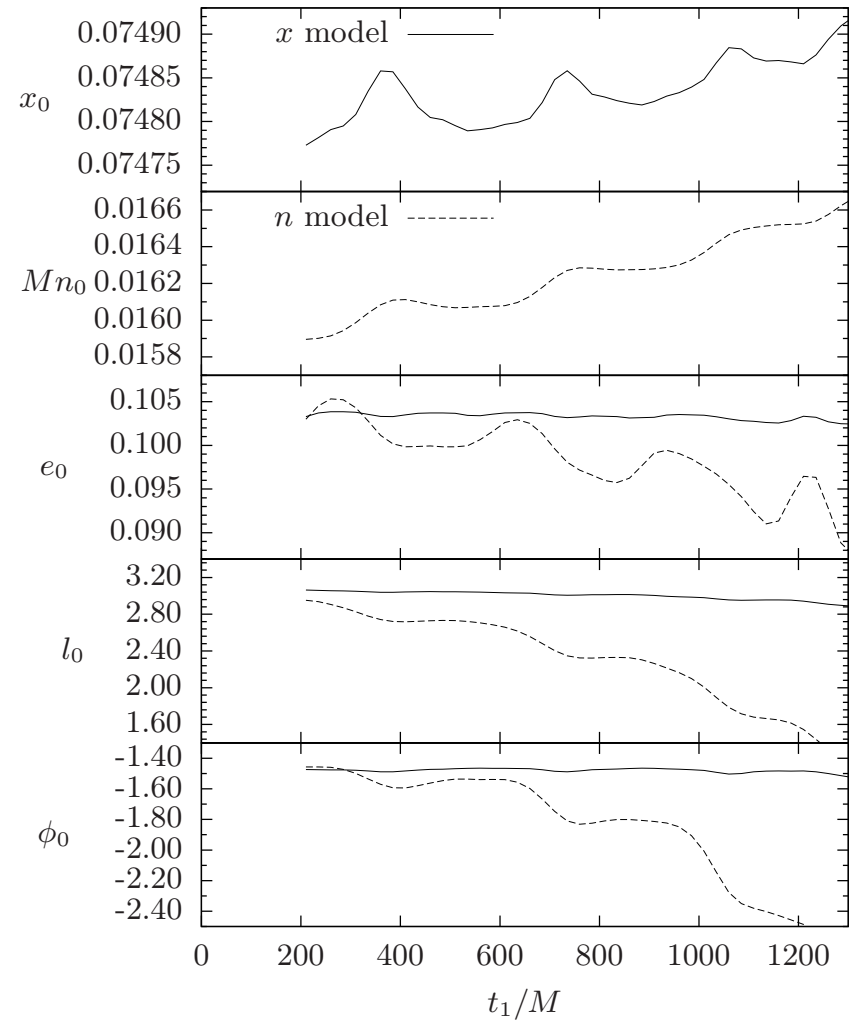

FIG. 5. PN parameters for the $x$ and $n$ models as determined from fitting windows $\left[t_{1}, t_{2}\right]$ for various values of $t_{1}$ and $t_{2}=$ $t_{1}+400 M$.

mined by fits of the NR data to the PN model in fitting intervals $I=\left[t_{1}, t_{2}\right]$. These parameters are the values of the functions $x, n, e, l$ and $\phi$ at $t-r^{\star}=0$. In Fig. 4 $t_{1}$ has been kept fixed to a value at the start of the usable waveform and $t_{2}$ has been varied. We see that the parameters obtained from fits using the $x$ model vary much less with the fitting window length than those using the $n$ model. Specifically, we see that for both models the fitted parameters oscillate significantly for intervals of less than $\sim 400 M$, but for the $x$ model these variations die away as the interval is increased beyond this. From the initial data parameters, the orbital period is $P=403 \mathrm{M}$. It may be that over timescales smaller then the orbital period, there are unmodeled non-adiabatic oscillations in the NR result which are averaged out when larger fitting intervals are used. These oscillations may cause the fit to become worse for small intervals. For the $n$ model we see strong oscillations of a period $\sim 400 M$ roughly corresponding to the period of the oscillations in $\omega_{\mathrm{gw}}$ itself. In order to determine the effect on the parameters of the interval location, we choose an interval width of $400 M$ and vary $t_{1}$ in Fig. 5. Here again we see that the $x$ model shows much more consistent behavior than the $n$ model.

In order to choose a unique set of PN parameters, we choose the earliest possible fitting interval, and take the 


\begin{tabular}{l|l|l|l}
\hline \hline Parameter & $x$-model fit & $n$-model fit & Initial data value \\
\hline$x_{0}$ & 0.0747729 & - & 0.0740853 \\
$n_{0}$ & - & 0.0158959 & 0.0156 \\
$e_{0}$ & 0.103291 & 0.10299 & 0.1 \\
$l_{0}$ & 3.06358 & 2.9529 & $\pi=3.1416$ \\
$\phi_{0}$ & -1.47386 & -1.45652 & 0 \\
\hline \hline
\end{tabular}

TABLE I. Eccentric PN ( $x$-model and $n$-model) parameters computed by fitting in an interval $[210,610]$ as well as the parameters estimated from the initial data. The parameters correspond to the values of the functions $x, n, e, l$ and $\phi$ at $t-r^{\star}=0$. Note that the agreement is not expected to be exact.

size of the interval to approximately correspond to the initial orbital period, $\sim 400 M$, giving a fitting interval $t / M \in[210,610]$. The parameters for this fitting interval are given in Table [. It is interesting to compare these parameters with the approximate parameters used to construct the Bowen-York initial data; these are also given in the table. $x_{0}$ and $n_{0}$ agree to within $1 \%$ and $2 \%$ respectively with the initial data values. $e_{0}$ agrees within $0.3 \%$ between the two PN models, and to $3 \%$ with the initial data value. $l_{0}$ agrees to within 0.1 radians between the two models and the initial data value. $\phi_{0}$ agrees to within 0.02 radians between the two models, but is of the order of $\pi / 2$ different from the initial data value. This large discrepancy is probably related to the adjustment of the coordinate system that happens at the start of the numerical simulation. Recall that the method for constructing the initial data parameters was approximate, due to the different coordinate systems used, so perfect agreement is not expected.

Now that we have estimated the PN model which matches the NR solution in the fitting interval, we can compare the PN waveform for the $x$ and $n$ models with the NR result. In Fig. 6 we plot the PN and NR gravitational wave frequencies $\omega_{\mathrm{gw}}$ and see that there is good agreement with the $x$ model from the start of the simulation to $t \approx 1800 M$. That there is such a high level of agreement with a model which contains so much structure is a strong validation of both the PN model and the NR simulation. We also see on the same plot the much worse agreement obtained using the $n$-model.

We now quantify the agreement with the $x$ and $n$ models by considering the waveform phase differences. Fig. 7 shows the difference between the NR and PN gravitational wave phases as a function of $t$. The error bars represent the uncertainty in the NR phase from extrapolation to infinite radius and finite differencing truncation error. We see that the phase difference between NR and $\mathrm{PN}$ is within 0.1 radians for approximately $1330 \mathrm{M}$, or $11 \mathrm{GW}$ cycles. At $t=1882 \mathrm{M}$, corresponding to $M \omega_{\mathrm{gw}}=0.1$, the phase difference between NR and PN is $\approx 0.7$ radians.

To put the phase difference of 0.7 radians at $M \omega_{\mathrm{gw}}=$

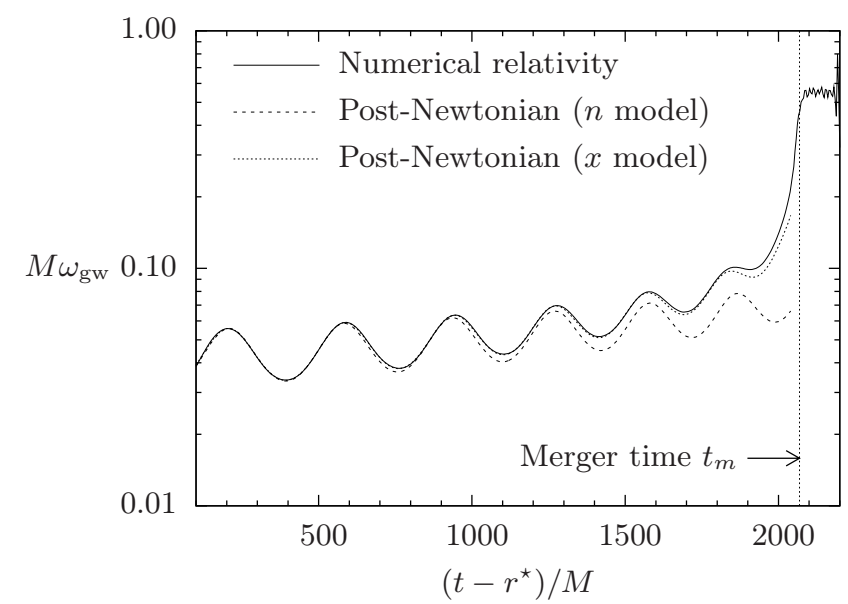

FIG. 6. Gravitational wave frequency as a function of time from the NR simulation and two PN models. The PN $x$-model agrees very well up to $\approx 1800 M$, whereas the agreement with the $n$-model is significantly worse.

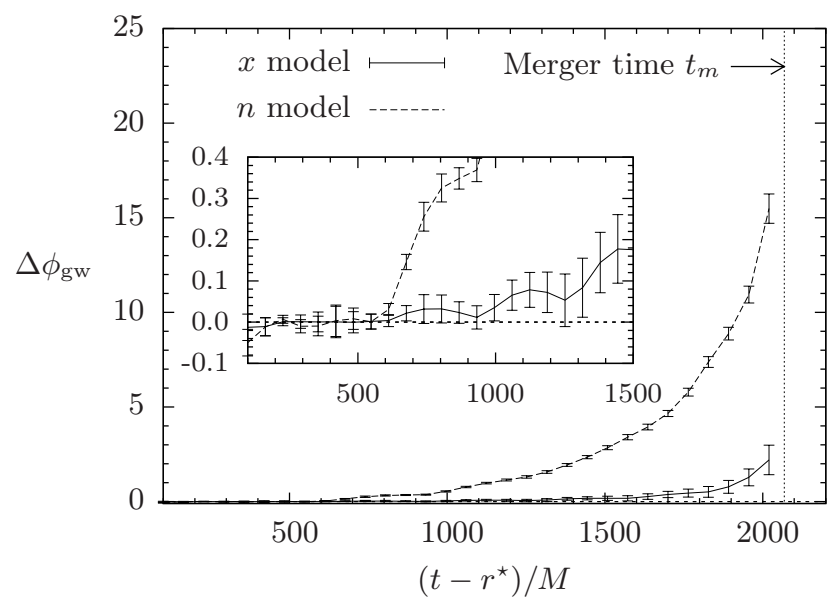

FIG. 7. Difference in gravitational wave phase between the NR simulation and the PN $x$-model. The error bars represent the estimated errors in the NR simulation.

0.1 into context, we note that the TaylorT4 circular PN model, which is very similar to our eccentric model with $e=0$, has been shown to have a phase difference at $M \omega_{\mathrm{gw}}=0.1$ of $\sim 0.3$ radians for $2 \mathrm{PN}$ radiation reaction (see Fig. 22 in Ref. 15]). We should be cautious about drawing the conclusion that the agreement in the circular case is better, however, as $M \omega_{\mathrm{gw}}=0.1$ may not be directly comparable in the two cases, particularly because $\omega_{\mathrm{gw}}$ oscillates in the eccentric case, but is monotonic in the circular case. The steepness of the phase difference in Fig. 22 in Ref. [15] at that point makes the comparison very sensitive to the exact point chosen. 


\section{Choice of post-Newtonian variables}

Throughout this work we have presented the results of fitting two PN models with NR data. The two models differ only in the choice of variable used: the frequencyrelated variable $x$ or the mean motion $n$. Our first attempts at matching the NR simulation with an eccentric PN model used $n$. We studied this case extensively, but found significant disagreement, as has been shown. Faced with this disagreement, we studied the (much simpler) circular case using a simulation 25] with low-eccentricity initial data [71] and a circular PN model formed by taking our eccentric $n$-model and setting $e=0$. This model is suboptimal as it only has $2 \mathrm{PN}$ radiation reaction, and 3.5 PN expressions are available for the circular case. The agreement between NR and PN is very poor even in the circular case using $n$; the gravitational wave phase difference at $M \omega_{\mathrm{gw}}=0.1$ is $\sim 20$ radians. (Note that one should be careful about making direct detailed comparisons between the circular and eccentric cases, due to the ambiguity in the choice of reference point $M \omega_{\mathrm{gw}}=0.1$ due to the eccentric oscillations in $\omega_{\mathrm{gw}}$.) However, expressing the PN equations in terms of the coordinate angular velocity of the black holes, $\omega$, as is common in the literature, gives a significant improvement over using $n$; at $M \omega_{\mathrm{gw}}=0.1$, the phase difference is 0.8 radians. This is in broad agreement with the difference of $\sim 0.3$ radians in Fig. 22 of Ref. 15] for the TaylorT4 model at 2 PN, accounting for the uncertainty in the choice of comparison time. This motivated us to search for a frequency-related variable applicable in the eccentric case, and we chose to use $x=(M \omega)^{2 / 3}$, for compatibility with Ref. [49] (recall that in the eccentric case, $\omega \equiv(2 \pi+\Delta \phi) / P \neq \dot{\phi})$, leading to the 0.7 radian phase difference at $M \omega_{\mathrm{gw}}=0.1$ we report here.

\section{CONCLUSIONS}

We have presented NR results for an inspiraling eccentric black hole binary system with initial eccentricity $e \approx 0.1$ and compared them with two adiabatic eccentric $\mathrm{PN}$ models $(x$ and $n$ ) with $2 \mathrm{PN}$ radiation reaction. For the $x$ model, the gravitational wave phase agrees to within \pm 0.1 radians between 21 and 11 cycles before merger. The difference grows to 0.7 radians at $\approx 5$ cycles before merger $\left(M \omega_{\mathrm{gw}}=0.1\right)$, in broad agreement with the circular case at $2 \mathrm{PN}$ order. One cycle before the merger, the solution to the PN ODEs diverges, indicating a breakdown of the model.

We found that it was necessary to express the PN model in terms of the frequency-related variable $x$ rather than the mean motion $n$ to get this level of agreement. We conjecture that, when expressed in terms of $n$, certain higher order PN terms are non-negligible, whereas when expressed in terms of $x$, they are small, leading to a smaller error in the PN solution. This can be likened to studies [13, 15] where different circular PN approximants of the same order have been shown to have different errors in the NR regime. In particular, the TaylorT4 circular model showed a remarkable agreement in the waveform phase, but there was a noticeable disagreement in the energy flux [17]. It has also been shown that this remarkable agreement is lost when spinning systems are considered [13]. Our eccentric PN model based on $x$ is very similar to TaylorT4 as $e \rightarrow 0$, so we would expect the same conclusions to apply.

Now that it is possible to match NR and PN eccentric waveforms, we plan to start to construct hybrid templates and begin to assess the implications for the interferometric detection of gravitational wave signals from eccentric binaries close to and including merger. Since complete $3 \mathrm{PN}$ radiation reaction terms for the angular momentum flux have now also been computed, we will be able to compare with a fully $3 \mathrm{PN}$ model, and expect the agreement with NR to get better closer to the merger.

\section{ACKNOWLEDGMENTS}

This work was supported in part by NSF grants PHY0925345 to DS, PHY-0653303, PHY-0555436, PHY0855892, PHY-0914553 to PL, PHY-0941417, PHY0903973 to PL and DS, and PHY-0114375 (CGWP). Computations were performed at NCSA and TACC under allocation TG-PHY060013N. Computer algebra and data analysis were performed using Mathematica. The authors thank M. Ansorg, T. Bode, A. Knapp, and E. Schnetter for contributions to the computational infrastructure and E. Bentivegna, J. Read and N. Yunes for helpful discussions. 


\section{Appendix A: PN expressions}

We now present, for reference, the full PN expressions used in this work. The expressions for the 3 PN conservative dynamics (i.e. $r, \dot{\phi}, l, n$ ) can be derived in two ways from the existing literature. They are given directly in Ref. [47] in terms of $n$ and $e_{t}$, so all that remains is to express them in terms of $x$ and $e_{t}$. Recall that $x$ is defined as $x \equiv(M \omega)^{2 / 3}$ where $\omega \equiv(2 \pi+\Delta \phi) / P$ and $P=2 \pi / n$. In Ref. [48], $\Phi$ is used in place of $\Delta \phi$, where $\Phi=2 \pi+\Delta \phi$. This reference gives expressions for $n, e_{t}$ and $\Phi$ in terms of $E$ and $J$; these can be used to obtain $n$ in terms of $x$ and $e_{t}$,

$$
\begin{aligned}
M n= & x^{3 / 2}+n_{1 \mathrm{PN}} x^{5 / 2}+n_{2 \mathrm{PN}} x^{7 / 2}+n_{3 \mathrm{PN}} x^{9 / 2}+\mathcal{O}\left(x^{11 / 2}\right) \\
n_{1 \mathrm{PN}}= & \frac{3}{e^{2}-1} \\
n_{2 \mathrm{PN}}= & \frac{(26 \eta-51) e^{2}+28 \eta-18}{4\left(e^{2}-1\right)^{2}} \\
n_{3 \mathrm{PN}}= & \frac{-1}{128\left(1-e^{2}\right)^{7 / 2}}\left[(1536 \eta-3840) e^{4}+(1920-768 \eta) e^{2}-768 \eta+\sqrt{1-e^{2}}\left(\left(1040 \eta^{2}-1760 \eta+2496\right) e^{4}\right.\right. \\
& \left.\left.+\left(5120 \eta^{2}+123 \pi^{2} \eta-17856 \eta+8544\right) e^{2}+896 \eta^{2}-14624 \eta+492 \eta \pi^{2}-192\right)+1920\right],
\end{aligned}
$$

where, for brevity, we have written $e \equiv e_{t}$. This expression for $n$ is then substituted into the conservative expressions in Ref. 47] to obtain the conservative expressions in terms of $x$ and $e_{t}$, dropping any resulting terms which are higher than 3 PN. Alternatively, we can derive these expressions by taking the expressions for the orbital elements in Ref. [48], along with the expressions for $r$ and $\dot{\phi}$, all in terms of $E$ and $J$. By both methods, we obtain for the separation $r$,

$$
\begin{aligned}
& r / M=r_{0 \mathrm{PN}} x^{-1}+r_{1 \mathrm{PN}}+r_{2 \mathrm{PN}} x+r_{3 \mathrm{PN}} x^{2}+\mathcal{O}\left(x^{3}\right) \\
& r_{\mathrm{OPN}}=1-e \cos (u) \\
& r_{1 \mathrm{PN}}=\frac{2(e \cos (u)-1)}{e^{2}-1}+\frac{1}{6}(2(\eta-9)+e(7 \eta-6) \cos (u)) \\
& r_{2 \mathrm{PN}}=\frac{1}{\left(1-e^{2}\right)^{2}}\left[\frac{1}{72}\left(8 \eta^{2}+30 \eta+72\right) e^{4}+\frac{1}{72}\left(-16 \eta^{2}-876 \eta+756\right) e^{2}+\frac{1}{72}\left(8 \eta^{2}+198 \eta+360\right)\right. \\
& +\left(\frac{1}{72}\left(-35 \eta^{2}+231 \eta-72\right) e^{5}+\frac{1}{72}\left(70 \eta^{2}-150 \eta-468\right) e^{3}+\frac{1}{72}\left(-35 \eta^{2}+567 \eta-648\right) e\right) \cos (u) \\
& \left.+\sqrt{1-e^{2}}\left(\frac{1}{72}(360-144 \eta) e^{2}+\frac{1}{72}(144 \eta-360)+\left(\frac{1}{72}(180-72 \eta) e^{3}+\frac{1}{72}(72 \eta-180) e\right) \cos (u)\right)\right] \\
& r_{3 \mathrm{PN}}=\frac{1}{181440\left(1-e^{2}\right)^{7 / 2}}\left[\left(-665280 \eta^{2}+1753920 \eta-1814400\right) e^{6}+\left(725760 \eta^{2}-77490 \pi^{2} \eta+5523840 \eta\right.\right. \\
& -3628800) e^{4}+\left(544320 \eta^{2}+154980 \pi^{2} \eta-14132160 \eta+7257600\right) e^{2}-604800 \eta^{2}+6854400 \eta \\
& +\left(\left(302400 \eta^{2}-1254960 \eta+453600\right) e^{7}+\left(-1542240 \eta^{2}-38745 \pi^{2} \eta+6980400 \eta-453600\right) e^{5}\right. \\
& +\left(2177280 \eta^{2}+77490 \pi^{2} \eta-12373200 \eta+4989600\right) e^{3}+\left(-937440 \eta^{2}-38745 \pi^{2} \eta+6647760 \eta\right. \\
& -4989600) e) \cos (u)+\sqrt{1-e^{2}}\left(\left(-4480 \eta^{3}-25200 \eta^{2}+22680 \eta-120960\right) e^{6}+\left(13440 \eta^{3}+4404960 \eta^{2}\right.\right. \\
& \left.+116235 \pi^{2} \eta-12718296 \eta+5261760\right) e^{4}+\left(-13440 \eta^{3}+2242800 \eta^{2}+348705 \pi^{2} \eta-19225080 \eta\right. \\
& +16148160) e^{2}+4480 \eta^{3}+45360 \eta^{2}-8600904 \eta+\left(\left(-6860 \eta^{3}+550620 \eta^{2}-986580 \eta+120960\right) e^{7}\right. \\
& +\left(20580 \eta^{3}-2458260 \eta^{2}+3458700 \eta-2358720\right) e^{5}+\left(-20580 \eta^{3}-3539340 \eta^{2}-116235 \pi^{2} \eta+20173860 \eta\right. \\
& \left.-16148160) e^{3}+\left(6860 \eta^{3}-1220940 \eta^{2}-464940 \pi^{2} \eta+17875620 \eta-4717440\right) e\right) \cos (u)+116235 \eta \pi^{2} \\
& \left.+1814400)-77490 \eta \pi^{2}-1814400\right] \text {. }
\end{aligned}
$$


The relative angular velocity $\dot{\phi}$ is found to be

$$
\begin{aligned}
M \dot{\phi}= & \dot{\phi}_{0 \mathrm{PN}} x^{3 / 2}+\dot{\phi}_{1 \mathrm{PN}} x^{5 / 2}+\dot{\phi}_{2 \mathrm{PN}} x^{7 / 2}+\dot{\phi}_{3 \mathrm{PN}} x^{9 / 2}+\mathcal{O}\left(x^{11 / 2}\right) \\
\dot{\phi}_{0 \mathrm{PN}}= & \frac{\sqrt{1-e^{2}}}{(e \cos (u)-1)^{2}} \\
\dot{\phi}_{1 \mathrm{PN}}= & -\frac{e(\eta-4)(e-\cos (u))}{\sqrt{1-e^{2}}(e \cos (u)-1)^{3}} \\
\dot{\phi}_{2 \mathrm{PN}}= & \frac{1}{12\left(1-e^{2}\right)^{3 / 2}(e \cos (u)-1)^{5}}\left[\left(-12 \eta^{2}-18 \eta\right) e^{6}+\left(20 \eta^{2}-26 \eta-60\right) e^{4}+\left(-2 \eta^{2}+50 \eta+75\right) e^{2}+\left[\left(-14 \eta^{2}\right.\right.\right. \\
& \left.+8 \eta-147) e^{5}+\left(8 \eta^{2}+22 \eta+42\right) e^{3}\right] \cos ^{3}(u)+\left[\left(17 \eta^{2}-17 \eta+48\right) e^{6}+\left(-4 \eta^{2}-38 \eta+153\right) e^{4}+\left(5 \eta^{2}-35 \eta\right.\right. \\
& \left.+114) e^{2}\right] \cos ^{2}(u)-36 \eta+\left[\left(-\eta^{2}+97 \eta+12\right) e^{5}+\left(-16 \eta^{2}-74 \eta-81\right) e^{3}+\left(-\eta^{2}+67 \eta-246\right) e\right] \cos (u) \\
& +\sqrt{1-e^{2}}\left[e^{3}(36 \eta-90) \cos ^{3}(u)+\left((180-72 \eta) e^{4}+(90-36 \eta) e^{2}\right) \cos { }^{2}(u)+\left((144 \eta-360) e^{3}\right.\right. \\
& \left.\left.+(90-36 \eta) e) \cos (u)+e^{2}(180-72 \eta)+36 \eta-90\right]+90\right]
\end{aligned}
$$

$$
\begin{aligned}
\dot{\phi}_{3 \mathrm{PN}}= & \frac{1}{13440\left(1-e^{2}\right)^{5 / 2}(e \cos (u)-1)^{7}}\left[\left(10080 \eta^{3}+40320 \eta^{2}-15120 \eta\right) e^{10}+\left(-52640 \eta^{3}-13440 \eta^{2}+483280 \eta\right) e^{8}\right. \\
& +\left(84000 \eta^{3}-190400 \eta^{2}-17220 \pi^{2} \eta-50048 \eta-241920\right) e^{6}+\left(-52640 \eta^{3}+516880 \eta^{2}+68880 \pi^{2} \eta\right.
\end{aligned}
$$$$
-1916048 \eta+262080) e^{4}+\left(4480 \eta^{3}-412160 \eta^{2}-30135 \pi^{2} \eta+553008 \eta+342720\right) e^{2}+\left(\left(13440 \eta^{3}+94640 \eta^{2}\right.\right.
$$$$
-113680 \eta-221760) e^{9}+\left(-11200 \eta^{3}-112000 \eta^{2}+12915 \pi^{2} \eta+692928 \eta-194880\right) e^{7}+\left(4480 \eta^{3}+8960 \eta^{2}\right.
$$$$
\left.\left.-43050 \pi^{2} \eta+1127280 \eta-147840\right) e^{5}\right) \cos ^{5}(u)+\left(\left(-16240 \eta^{3}+12880 \eta^{2}+18480 \eta\right) e^{10}+\left(16240 \eta^{3}-91840 \eta^{2}\right.\right.
$$$$
\left.+17220 \pi^{2} \eta-652192 \eta+100800\right) e^{8}+\left(-55440 \eta^{3}+34160 \eta^{2}-30135 \pi^{2} \eta-2185040 \eta+2493120\right) e^{6}
$$$$
\left.+\left(21840 \eta^{3}+86800 \eta^{2}+163590 \pi^{2} \eta-5713888 \eta+228480\right) e^{4}\right) \cos ^{4}(u)+\left(\left(560 \eta^{3}-137200 \eta^{2}+388640 \eta\right.\right.
$$$$
+241920) e^{9}+\left(30800 \eta^{3}-264880 \eta^{2}-68880 \pi^{2} \eta+624128 \eta+766080\right) e^{7}+\left(66640 \eta^{3}+612080 \eta^{2}-8610 \pi^{2} \eta\right.
$$$$
\left.+6666080 \eta-6652800) e^{5}+\left(-30800 \eta^{3}-294000 \eta^{2}-223860 \pi^{2} \eta+9386432 \eta\right) e^{3}\right) \cos ^{3}(u)+67200 \eta^{2}
$$$$
+\left(\left(4480 \eta^{3}-20160 \eta^{2}+16800 \eta\right) e^{10}+\left(3920 \eta^{3}+475440 \eta^{2}-17220 \pi^{2} \eta+831952 \eta-725760\right) e^{8}+\left(-75600 \eta^{3}\right.\right.
$$$$
\left.+96880 \eta^{2}+154980 \pi^{2} \eta-3249488 \eta-685440\right) e^{6}+\left(5040 \eta^{3}-659120 \eta^{2}+25830 \pi^{2} \eta-7356624 \eta+6948480\right) e^{4}
$$$$
\left.+\left(-5040 \eta^{3}+190960 \eta^{2}+137760 \pi^{2} \eta-7307920 \eta+107520\right) e^{2}\right) \cos ^{2}(u)-761600 \eta+\left(\left(-2240 \eta^{3}-168000 \eta^{2}\right.\right.
$$$$
-424480 \eta) e^{9}+\left(28560 \eta^{3}+242480 \eta^{2}+34440 \pi^{2} \eta-1340224 \eta+725760\right) e^{7}+\left(-33040 \eta^{3}-754880 \eta^{2}\right.
$$$$
\left.-172200 \pi^{2} \eta+5458480 \eta-221760\right) e^{5}+\left(40880 \eta^{3}+738640 \eta^{2}+30135 \pi^{2} \eta+1554048 \eta-2936640\right) e^{3}
$$$$
\left.+\left(-560 \eta^{3}-100240 \eta^{2}-43050 \pi^{2} \eta+3284816 \eta-389760\right) e\right) \cos (u)+\sqrt{1-e^{2}}\left(\left(\left(-127680 \eta^{2}+544320 \eta\right.\right.\right.
$$$$
\left.-739200) e^{7}+\left(-53760 \eta^{2}-8610 \pi^{2} \eta+674240 \eta-67200\right) e^{5}\right) \cos ^{5}(u)+\left(\left(161280 \eta^{2}-477120 \eta+537600\right) e^{8}\right.
$$$$
+\left(477120 \eta^{2}+17220 \pi^{2} \eta-2894080 \eta+2217600\right) e^{6}+\left(268800 \eta^{2}+25830 \pi^{2} \eta-2721600 \eta\right.
$$$$
\left.+1276800) e^{4}\right) \cos ^{4}(u)+\left(\left(-524160 \eta^{2}+1122240 \eta-940800\right) e^{7}+\left(-873600 \eta^{2}-68880 \pi^{2} \eta+7705600 \eta\right.\right.
$$$$
\left.-3897600) e^{5}+\left(-416640 \eta^{2}-17220 \pi^{2} \eta+3357760 \eta-3225600\right) e^{3}\right) \cos ^{3}(u)+\left(\left(604800 \eta^{2}-504000 \eta\right.\right.
$$$$
-403200) e^{6}+\left(1034880 \eta^{2}+103320 \pi^{2} \eta-11195520 \eta+5779200\right) e^{4}+\left(174720 \eta^{2}-17220 \pi^{2} \eta-486080 \eta\right.
$$$$
\left.+2688000) e^{2}\right) \cos ^{2}(u)+\left(\left(-282240 \eta^{2}-450240 \eta+1478400\right) e^{5}+\left(-719040 \eta^{2}-68880 \pi^{2} \eta+8128960 \eta\right.\right.
$$$$
\left.-5040000) e^{3}+\left(94080 \eta^{2}+25830 \pi^{2} \eta-1585920 \eta-470400\right) e\right) \cos (u)-67200 \eta^{2}+761600 \eta+e^{4}\left(40320 \eta^{2}\right.
$$$$
\left.+309120 \eta-672000)+e^{2}\left(208320 \eta^{2}+17220 \pi^{2} \eta-2289280 \eta+1680000\right)-8610 \eta \pi^{2}-201600\right)+8610 \eta \pi^{2}
$$$$
+201600] \text {. }
$$ 
The $3 \mathrm{PN}$ Kepler equation is

$$
\begin{aligned}
l= & l_{0 \mathrm{PN}}+l_{2 \mathrm{PN}} x^{2}+l_{3 \mathrm{PN}} x^{3}+\mathcal{O}\left(x^{4}\right) \\
l_{0 \mathrm{PN}}= & u-e \sin u \\
l_{2 \mathrm{PN}}= & \frac{1}{8 \sqrt{1-e^{2}}(1-e \cos (u))}\left[-12(2 \eta-5)(u-v)(e \cos (u)-1)-e \sqrt{1-e^{2}}(\eta-15) \eta \sin (u)\right] \\
l_{3 \mathrm{PN}}= & \frac{1}{6720\left(1-e^{2}\right)^{3 / 2}(1-e \cos (u))^{3}}\left[3 5 \left(96\left(11 \eta^{2}-29 \eta+30\right) e^{2}+960 \eta^{2}+\eta\left(-13184+123 \pi^{2}\right)\right.\right. \\
& +8640)(u-v)(e \cos (u)-1)^{3}+3360(-12(2 \eta-5)(u-v)+12 e(2 \eta-5) \cos (u)(u-v) \\
& \left.+e \sqrt{1-e^{2}}(\eta-15) \eta \sin (u)\right)(e \cos (u)-1)^{2}+e \sqrt{1-e^{2}}\left(140\left(13 e^{4}-11 e^{2}-2\right) \eta^{3}-140\left(73 e^{4}-325 e^{2}+444\right) \eta^{2}\right. \\
& +\left(3220 e^{4}-148960 e^{2}-4305 \pi^{2}+143868\right) \eta+e^{2}\left(1820\left(e^{2}-1\right) \eta^{3}-140\left(83 e^{2}+109\right) \eta^{2}-\left(1120 e^{2}+4305 \pi^{2}\right.\right. \\
& +752) \eta+67200) \cos ^{2}(u)-2 e\left(1960\left(e^{2}-1\right) \eta^{3}+6720\left(e^{2}-5\right) \eta^{2}+\left(-71820 e^{2}-4305 \pi^{2}+69948\right) \eta\right. \\
& +67200) \cos (u)+67200) \sin (u)]
\end{aligned}
$$

where, as in Ref. [47], we use

$$
v-u=2 \tan ^{-1}\left(\frac{\sin (u) \beta_{\phi}}{1-\cos (u) \beta_{\phi}}\right)
$$

and

$$
\beta_{\phi}=\frac{1-\sqrt{1-e_{\phi}^{2}}}{e_{\phi}}
$$

$e_{\phi}$ is given by

$$
\begin{aligned}
e_{\phi}= & e+e_{\phi 1 \mathrm{PN}} x+e_{\phi 2 \mathrm{PN}} x^{2}+e_{\phi 3 \mathrm{PN}} x^{3}+\mathcal{O}\left(x^{4}\right) \\
e_{\phi 1 \mathrm{PN}}= & -e(\eta-4) \\
e_{\phi 2 \mathrm{PN}}= & \frac{e}{96\left(e^{2}-1\right)}\left[\left(41 \eta^{2}-659 \eta+1152\right) e^{2}+4 \eta^{2}+68 \eta+\sqrt{1-e^{2}}(288 \eta-720)-1248\right] \\
e_{\phi 3 \mathrm{PN}}= & -\frac{e}{26880\left(1-e^{2}\right)^{5 / 2}}\left[\left(13440 \eta^{2}+483840 \eta-940800\right) e^{4}+\left(255360 \eta^{2}+17220 \pi^{2} \eta-2880640 \eta+2688000\right) e^{2}\right. \\
& -268800 \eta^{2}+2396800 \eta+\sqrt{1-e^{2}}\left(\left(1050 \eta^{3}-134050 \eta^{2}+786310 \eta-860160\right) e^{4}+\left(-18900 \eta^{3}+553980 \eta^{2}\right.\right. \\
& \left.\left.+4305 \pi^{2} \eta-1246368 \eta+2042880\right) e^{2}+276640 \eta^{2}+2674480 \eta-17220 \eta \pi^{2}-1451520\right)-17220 \eta \pi^{2} \\
& -1747200] .
\end{aligned}
$$

This completes the expressions used in the conservative dynamics. The radiation reaction is given to $2 \mathrm{PN}$ order in Ref. [47] in terms of $n$ and $e_{t}$. We again substitute for $n$ in terms of $x$, and obtain

$$
\begin{aligned}
M \dot{x}= & \dot{x}_{0 \mathrm{PN}} x^{5}+\dot{x}_{1 \mathrm{PN}} x^{6}+\dot{x}_{1.5 \mathrm{PN}} x^{13 / 2}+\dot{x}_{2 \mathrm{PN}} x^{7}+\mathcal{O}\left(x^{15 / 2}\right) \\
\dot{x}_{0 \mathrm{PN}}= & \frac{2\left(37 e^{4}+292 e^{2}+96\right) \eta}{15\left(1-e^{2}\right)^{7 / 2}} \\
\dot{x}_{1 \mathrm{PN}}= & \frac{\eta}{420\left(1-e^{2}\right)^{9 / 2}}\left[-(8288 \eta-11717) e^{6}-14(10122 \eta-12217) e^{4}-120(1330 \eta-731) e^{2}-16(924 \eta+743)\right] \\
\dot{x}_{1.5 \mathrm{PN}}= & \frac{256}{5} \eta \pi \kappa_{E}(e) \\
\dot{x}_{2 \mathrm{PN}=} & \frac{\eta}{45360\left(1-e^{2}\right)^{11 / 2}}\left[\left(1964256 \eta^{2}-3259980 \eta+3523113\right) e^{8}+\left(64828848 \eta^{2}-123108426 \eta+83424402\right) e^{6}\right. \\
& +\left(16650606060 \eta^{2}-207204264 \eta+783768\right) e^{4}+\left(61282032 \eta^{2}+15464736 \eta-92846560\right) e^{2}+1903104 \eta^{2} \\
& +\sqrt{1-e^{2}}\left((2646000-1058400 \eta) e^{6}+(64532160-25812864 \eta) e^{2}-580608 \eta+1451520\right)+4514976 \eta \\
& -360224] \\
&
\end{aligned}
$$


for $\dot{x}$, and

$$
\begin{aligned}
M \dot{e}= & \dot{e}_{0 \mathrm{PN}} x^{4}+\dot{e}_{1 \mathrm{PN}} x^{5}+\dot{e}_{1.5 \mathrm{PN}} x^{11 / 2}+\dot{e}_{2 \mathrm{PN}} x^{6}+\mathcal{O}\left(x^{13 / 2}\right) \\
\dot{e}_{0 \mathrm{PN}}= & -\frac{e\left(121 e^{2}+304\right) \eta}{15\left(1-e^{2}\right)^{5 / 2}} \\
\dot{e}_{1 \mathrm{PN}}= & \frac{e \eta}{2520\left(1-e^{2}\right)^{7 / 2}}\left[(93184 \eta-125361) e^{4}+12(54271 \eta-59834) e^{2}+8(28588 \eta+8451)\right] \\
\dot{e}_{1.5 \mathrm{PN}}= & \frac{128 \eta \pi}{5 e}\left[\left(e^{2}-1\right) \kappa_{E}(e)+\sqrt{1-e^{2}} \kappa_{J}(e)\right] \\
\dot{e}_{2 \mathrm{PN}}= & -\frac{e \eta}{30240\left(1-e^{2}\right)^{9 / 2}}\left[\left(2758560 \eta^{2}-4344852 \eta+3786543\right) e^{6}+\left(42810096 \eta^{2}-78112266 \eta+46579718\right) e^{4}\right. \\
& +\left(48711348 \eta^{2}-35583228 \eta-36993396\right) e^{2}+4548096 \eta^{2}+\sqrt{1-e^{2}}\left((2847600-1139040 \eta) e^{4}+(35093520\right. \\
& \left.\left.-14037408 \eta) e^{2}-5386752 \eta+13466880\right)+13509360 \eta-15198032\right],
\end{aligned}
$$

for $\dot{e}$. These equations are written in terms of the functions $\kappa_{E}$ and $\kappa_{J}$, given in Ref. [46] in terms of infinite sums of Bessel functions. We reproduce them here for completeness.

$$
\begin{aligned}
\kappa_{E}= & \sum_{p=1}^{\infty} \frac{1}{4} p^{3}\left(\left(\left(-e^{2}-\frac{3}{e^{2}}+\frac{1}{e^{4}}+3\right) p^{2}+\frac{1}{3}-\frac{1}{e^{2}}+\frac{1}{e^{4}}\right) J_{p}(p e)^{2}+\left(-3 e-\frac{4}{e^{3}}+\frac{7}{e}\right) p J_{p}^{\prime}(p e) J_{p}(p e)\right. \\
& \left.+\left(\left(e^{2}+\frac{1}{e^{2}}-2\right) p^{2}+\frac{1}{e^{2}}-1\right) J_{p}^{\prime}(p e)^{2}\right) \\
\kappa_{J}= & \sum_{p=1}^{\infty} \frac{1}{2} p^{2} \sqrt{1-e^{2}}\left(\left(-\frac{2}{e^{4}}-1+\frac{3}{e^{2}}\right) p J_{p}(p e)^{2}+\left(2\left(e+\frac{1}{e^{3}}-\frac{2}{e}\right) p^{2}-\frac{1}{e}+\frac{2}{e^{3}}\right) J_{p}^{\prime}(p e) J_{p}(p e)+2\left(1-\frac{1}{e^{2}}\right) p J_{p}^{\prime}(p e)^{2}\right)
\end{aligned}
$$

These are functions of $e$ only, and are computed numerically using a sufficient number of terms in the summation that the result converges to within machine precision $\left(10^{-15}\right)$. For computational efficiency, the resulting function is converted into an interpolating polynomial, and the interpolation error is estimated to be $\sim 10^{-12}$ in the range $0<e \leq 0.4$.

[1] B. C. Barish and R. Weiss, Phys. Today 52N10, 44 (1999).

[2] S. J. Waldmann (LIGO Scientific), Class. Quant. Grav. 23, S653 (2006).

[3] S. Hild (LIGO Scientific), Class. Quant. Grav. 23, S643 (2006).

[4] F. Acernese et al. (VIRGO), Class. Quant. Grav. 19, 1421 (2002).

[5] F. Acernese et al., Class. Quant. Grav. 23, S635 (2006).

[6] N. Yunes and E. Berti, Phys. Rev. D77, 124006 (2008), arXiv:0803.1853 [gr-qc].

[7] F. Pretorius, Phys. Rev. Lett. 95, 121101 (Sep. 2005), gr-qc/0507014

[8] M. Campanelli, C. O. Lousto, P. Marronetti, and Y. Zlochower, Phys. Rev. Lett. 96, 111101 (2006), arXiv:gr-qc/0511048

[9] J. G. Baker, J. Centrella, D.-I. Choi, M. Koppitz, and J. van Meter, Phys. Rev. Lett. 96, 111102 (2006), arXiv:gr-qc/0511103.

[10] J. G. Baker, J. Centrella, D.-I. Choi, M. Koppitz, and J. van Meter, Phys. Rev. D73, 104002 (2006).

[11] A. Buonanno, G. B. Cook, and F. Pretorius, Phys. Rev. D 75, 124018 (Jun. 2007),
arXiv:gr-qc/0610122

[12] J. G. Baker, J. R. van Meter, S. T. McWilliams, J. Centrella, and B. J. Kelly, Phys. Rev. Lett. 99, 181101 (2007), arXiv:gr-qc/0612024

[13] M. Hannam, S. Husa, U. Sperhake, B. Brügmann, and J. A. González, Phys. Rev. D77, 044020 (2008), arXiv:0706.1305 [gr-qc].

[14] A. Gopakumar, M. Hannam, S. Husa, and B. Bruegmann, Phys. Rev. D78, 064026 (2008), arXiv:0712.3737 [gr-qc],

[15] M. Boyle et al., Phys. Rev. D76, 124038 (2007), arXiv:0710.0158 [gr-qc],

[16] T. Damour, A. Nagar, E. N. Dorband, D. Pollney, and L. Rezzolla, Phys. Rev. D77, 084017 (2008), arXiv:0712.3003 [gr-qc].

[17] M. Boyle et al.(2008), arXiv:0804.4184 [gr-qc].

[18] E. Berti et al., Phys. Rev. D76, 064034 (2007), arXiv:gr-qc/0703053.

[19] M. Hannam, S. Husa, B. Bruegmann, and A. Gopakumar, Phys. Rev. D78, 104007 (2008), arXiv:0712.3787 [gr-qc].

[20] P. Ajith et al., Phys. Rev. D77, 104017 (2008), arXiv:0710.2335 [gr-qc]. 
[21] P. Ajith, Class. Quant. Grav. 25, 114033 (2008), arXiv:0712.0343 [gr-qc].

[22] A. Buonanno et al., Phys. Rev. D76, 104049 (2007), arXiv:0706.3732 [gr-qc].

[23] Y. Pan et al., Phys. Rev. D77, 024014 (2008), arXiv:0704.1964 [gr-qc]

[24] U. Sperhake et al., Phys. Rev. D78, 064069 (2008), arXiv:0710.3823 [gr-qc].

[25] I. Hinder, B. Vaishnav, F. Herrmann, D. Shoemaker, and P. Laguna, Phys. Rev. D77, 081502 (2008), arXiv:0710.5167 [gr-qc].

[26] P. C. Peters, Phys. Rev. 136, B1224 (1964).

[27] M. Campanelli, C. O. Lousto, and Y. Zlochower, Phys. Rev. D77, 101501 (2008), arXiv:0710.0879 [gr-qc]

[28] Y. Kozai, Astronomical Journal 67, 591 (Nov. 1962).

[29] L. Wen, Astrophys. J. 598, 419 (Nov. 2003).

[30] M. J. Benacquista, Living Rev. Rel. 5, 2 (2002), arXiv:astro-ph/0202056

[31] K. Gultekin, M. C. Miller, and D. P. Hamilton, Astrophys. J. 616, 221 (2004), arXiv:astro-ph/0402532.

[32] K. S. Thorne and V. B. Braginskii, Astrophys. J. Lett. 204, L1 (Feb. 1976).

[33] O. Blaes, M. H. Lee, and A. Socrates, Astrophys. J. 578, 775 (2002), arXiv:astro-ph/0203370.

[34] M. Dotti, M. Colpi, and F. Haardt, Mon. Not. R. Astron. Soc. 367, 103 (Mar. 2006).

[35] P. C. Peters and J. Mathews, Phys. Rev. 131, 435 (Jul 1963).

[36] R. V. Wagoner and C. M. Will, Astrophys. J. 210, 764 (1976).

[37] L. Blanchet and G. Schäfer, Mon. Not. R. Astron. Soc. 239, 845 (1989).

[38] W. Junker and G. Schäfer, Mon. Not. R. Astron. Soc. 254, 146 (Jan. 1992).

[39] L. Blanchet and G. Schäfer, Class. Quant. Grav. 10, 2699 (1993).

[40] R. Rieth and G. Schäfer, Class. Quant. Grav. 14, 2357 (1997).

[41] T. Damour and G. Schäfer, Nuovo Cim. B101, 127 (1988).

[42] G. Schäfer and N. Wex, Physics Lett. A 174, 196 (1993).

[43] N. Wex, Classical and Quantum Gravity 12, 983 (Apr. 1995).

[44] A. Gopakumar and B. R. Iyer, Phys. Rev. D56, 7708 (1997), arXiv:gr-qc/9710075.

[45] A. Gopakumar and B. R. Iyer, Phys. Rev. D65, 084011 (2002), arXiv:gr-qc/0110100

[46] T. Damour, A. Gopakumar, and B. R. Iyer, Phys. Rev. D70, 064028 (2004), arXiv:gr-qc/0404128

[47] C. Königsdörffer and A. Gopakumar, Phys. Rev. D 73, 124012 (Jun. 2006).

[48] R.-M. Memmesheimer, A. Gopakumar, and G. Schäfer, Phys. Rev. D70, 104011 (2004), arXiv:gr-qc/0407049
[49] K. G. Arun, L. Blanchet, B. R. Iyer, and M. S. S. Qusailah, Phys. Rev. D 77, 064035 (Mar. 2008), arXiv:0711.0302

[50] K. G. Arun, L. Blanchet, B. R. Iyer, and M. S. S. Qusailah, Phys. Rev. D 77, 064034 (Mar. 2008), arXiv:0711.0250

[51] K. Arun, Ph.D. thesis, Jawaharlal Nehru University, New Delhi (2006).

[52] K. G. Arun, L. Blanchet, B. R. Iyer, and S. Sinha, Phys. Rev. D80, 124018 (2009), arXiv:0908.3854 [gr-qc]

[53] L. Blanchet, Living Reviews in Relativity 9, 4 (Jun. 2006).

[54] H. Goldstein, C. P. Poole, and J. L. Safko, Classical Mechanics (Addison-Wesley, 2002).

[55] J. N. Goldberg, A. J. MacFarlane, E. T. Newman, F. Rohrlich, and E. C. G. Sudarshan, J. Math. Phys. 8, 2155 (1967).

[56] C. M. Will and A. G. Wiseman, Phys. Rev. D 54, 4813 (1996), gr-qc/9608012.

[57] M. Ansorg, B. Brügmann, and W. Tichy, Phys. Rev. D 70, 064011 (2004).

[58] T. Nakamura, K. Oohara, and Y. Kojima, Prog. Theor. Phys. Suppl. 90, 1 (1987).

[59] M. Shibata and T. Nakamura, Phys. Rev. D 52, 5428 (1995).

[60] T. Baumgarte and S. Shapiro, Phys. Rev. D 59, 024007 (1999).

[61] S. Husa, I. Hinder, and C. Lechner, Computer Physics Communications 174, 983 (Jun. 2006).

[62] Cactus Computational Toolkit home page, http://www. cactuscode.org/.

[63] E. Schnetter, S. H. Hawley, and I. Hawke, Class. Quant. Grav. 21, 1465 (21 March 2004).

[64] B. Vaishnav, I. Hinder, F. Herrmann, and D. Shoemaker, Phys. Rev. D 76, 084020 (2007).

[65] S. Husa, J. A. Gonzalez, M. Hannam, B. Bruegmann, and U. Sperhake, Class. Quant. Grav. 25, 105006 (2008), arXiv:0706.0740 [gr-qc].

[66] M. Alcubierre, B. Brügmann, P. Diener, M. Koppitz, D. Pollney, E. Seidel, and R. Takahashi, Phys. Rev. D 67, 084023 (2003), gr-qc/0206072.

[67] D. R. Fiske, J. G. Baker, J. R. van Meter, D. Choi, and J. M. Centrella, Phys. Rev. D 71, 104036 (2005), grqc/0503100.

[68] T. Bode, D. Shoemaker, F. Herrmann, and I. Hinder, Phys. Rev. D77, 044027 (2008), arXiv:0711.0669 [gr-qc]

[69] F. Herrmann, I. Hinder, D. M. Shoemaker, P. Laguna, and R. A. Matzner, Phys. Rev. D76, 084032 (2007), arXiv:0706.2541 [gr-qc].

[70] A. Ashtekar and B. Krishnan, Living Rev. Rel. 7, 10 (2004).

[71] S. Husa, M. Hannam, J. A. González, U. Sperhake, and B. Brügmann, Phys. Rev. D77, 044037 (2008), arXiv:0706.0904 [gr-qc]. 